\title{
A Hybrid Procedure Combining Mini-Thoracotomy with Interventional Endocardial Lead Implantation for Cardiac Resynchronization Therapy in Patients with Chronic Congestive Heart Failure: A Report of Four Cases
}

\author{
Haiyan Xiang, Rifeng Gao, Juesheng Yang, Juxiang Li, Jin Li, Fei Lu, Yanhua Tang \\ Department of Cardiovascular Surgery, The Second Affiliated Hospital of Nanchang University, Nanchang University, Nanchang, \\ 330006, Jiangxi Province, China
}

\section{ABSTRACT}

Background: We describe the application and effectiveness of transthoracic electrode implantation for epicardial left ventricular pacing in cardiac resynchronization therapy (CRT) for patients with chronic congestive heart failure.

Methods: We assessed four patients with chronic congestive heart failure for whom implantation of endocardial electrodes was contraindicated. The epicardial electrodes were implanted via a mini-thoracotomy in the fourth or fifth left intercostal space. We analyzed the surgical implantation technique and the short-term effectiveness of the procedure.

Results: The epicardial electrodes successfully were implanted in all four patients. The patients' hemodynamic status, cardiac function, and symptoms significantly improved. Patients I, II, III, and IV were discharged from the hospital on the $8,11,4$, and 7 days, respectively, after the operation. Follow up lasted for 12 months. None of the patients presented with electrode fractures or surgical wound infections, and the pacing threshold and electrode impedance were normal. In one case, phrenic nerve stimulation occurred due to the low placement position of the electrode. When the electrode was moved slightly inward and upward, the sacral nerve stimulation sign disappeared, and no other complications were noted. One patient developed capsule infection, and the presence of an ectopic pacemaker was noted; therefore, a pacemaker replacement procedure was required.

Conclusion: In CRT, the implantation of a left ventricular epicardial electrode through a left-sided small incision is safe, feasible, and effective. This hybrid surgery combining interventional and cardiac techniques can maximize the curative effect of CRT.

\section{INTRODUCTION}

Chronic heart failure, also known as chronic congestive heart failure, is a commonly observed clinical syndrome

Received November 22, 2019; accepted April 29, 2020.

Correspondence: Yanhua Tang, Department of Cardiovascular Surgery, The Second Affiliated Hospital of Nanchang University, Fiangxi Province, China (e-mail: tyb6565@163.com).
[Brennan 2018; Drugs for Chronic Heart Failure 2019]. It has attracted attention from the medical community owing to the associated high morbidity and mortality rates. Cardiac resynchronization therapy (CRT) can improve the clinical symptoms of patients with chronic heart failure, enhance their exercise tolerance, and significantly reduce the hospitalization and mortality rates. The European Society of Cardiology in 2005 and the Chinese Medical Association in 2012 have included CRT as a class I indication of non-drug therapy in their treatment guidelines. Approximately 10-30\% of the patients reportedly are unable to undergo transvenous left ventricular lead implantation owing to congenital or acquired factors [Konstantinides 2020; Harjola 2018]. We performed combined epicardial left ventricular lead implantation via small chest incisions and interventional endocardial lead implantation during CRT in four patients with chronic congestive heart failure for whom left ventricular endocardial lead implantation was contraindicated. Herein, we describe the application and effectiveness of this technique. Specifically, we present the key technical points, intraoperative status, and postoperative follow-up results for these implantation procedures.

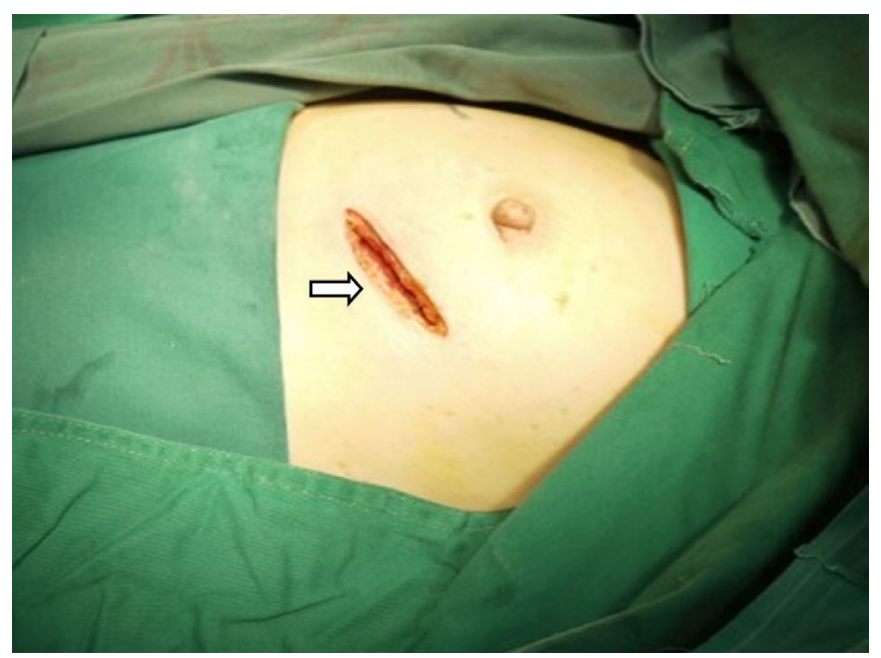

Figure 1. An incision of about $5 \mathrm{~cm}$ in length was made at the left anterolateral chest through the fifth intercostal space. 


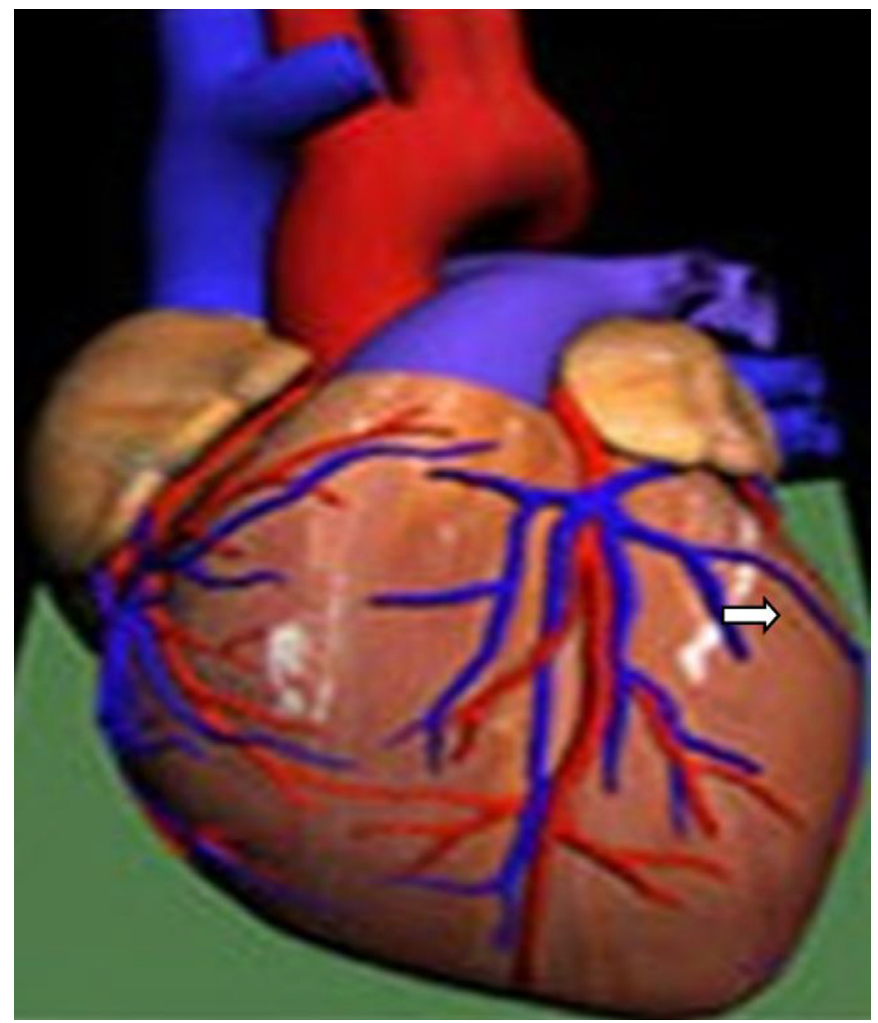

Figure 2. Two 6-0 prolene sutures were preset in the epicardium beneath the first diagonal branch of the left anterior descending artery to fix the epicardial lead in the avascular zone.

\section{CASE REPORT}

Case I: A 76-year-old female patient was admitted owing to repeated episodes of chest tightness and shortness of breath experienced for $>10$ years and chest pain experienced for one year, which had been aggravated for two weeks prior. The patient was diagnosed with dilated cardiomyopathy. Electrocardiography revealed a sinus rhythm, QRS length of $170 \mathrm{~ms}$, complete left bundle branch block, PR interval of $150 \mathrm{~ms}$, and P wave duration of $110 \mathrm{~ms}$. Echocardiography revealed that the left ventricular end diastolic diameter (LVEDD) was $83 \mathrm{~mm}$; left ventricular end systolic diameter (LVESD) was $70 \mathrm{~mm}$; and left ventricular ejection fraction (LVEF) $26 \%$. The cardiothoracic ratio was 0.70 . The clinical characteristics of the case were in accordance with the class I indication for CRT. Coronary sinus malformation and the great cardiac vein and posterior vein of the left ventricle were observed on angiography. Hence, transvenous implantation of the left ventricular lead was not possible. Therefore, we performed epicardial left ventricular lead implantation via a small chest incision.

The surgery was divided into two parts. First, right atrial and right ventricular lead implantation was performed in a catheterization laboratory. The patient then underwent epicardial left ventricular lead implantation in the operating room. The patient was placed in the supine position, and the left chest was elevated. An incision of approximately $5 \mathrm{~cm}$ in

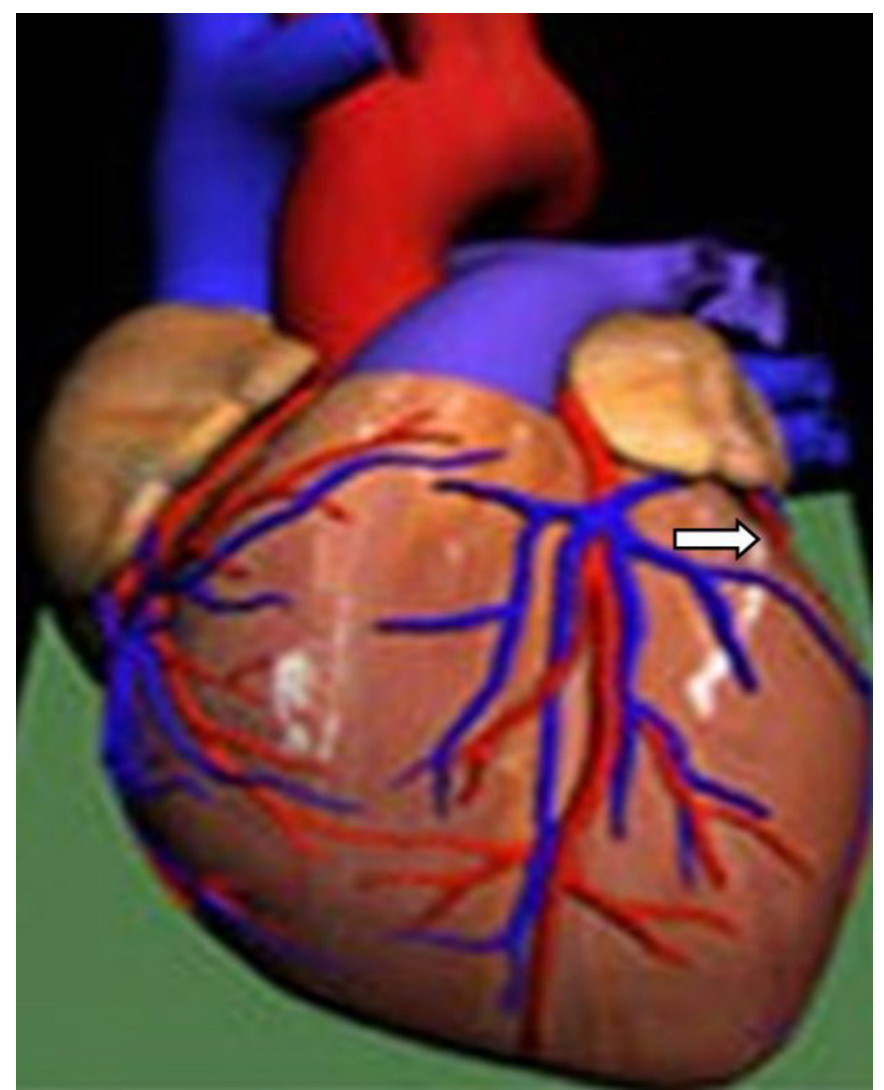

Figure 3. Two 6-0 prolene sutures were preset in the triangle region located inferior to the left atrioventricular groove and superior to the first obtuse marginal branch of the left circumflex artery, and the epicardial lead was fixed in this avascular zone.

length was created in the left anterolateral chest (Figure 1). The chest was accessed through the fifth intercostal space. A longitudinal incision was created in the anterior pericardium to expose the heart. Two 6-0 Prolene sutures were placed in the epicardium beneath the first diagonal branch of the left anterior descending artery to fix the epicardial lead in the avascular zone (Figure 2). The pacing parameters were measured. The left ventricular impedance was $783 \Omega$; threshold, $2.0 \mathrm{mV}$; and the R-wave amplitude, $2.5 \mathrm{mV}$. A small incision was then created made in the corresponding part of the pericardium to allow for the removal of the epicardial lead line, which was then pulled through the upper intercostal space to the subcutaneous tunnel and brought into the pocket of the pacemaker. A fistula was created in the pericardium posterior to the left phrenic nerve for drainage. Intermittent suturing of the pericardial incision was performed. The pacing parameters were measured again. The left ventricular impedance was $783 \Omega$; threshold, $1.0 \mathrm{~V}$; and R-wave amplitude, $2.5 \mathrm{mV}$. Phrenic nerve irritation, corresponding to the higher threshold, was observed once during the adjustment process. A drainage tube was placed through the left chest wall, and the chest wound was closed.

Case II: A 59-year-old female patient was admitted owing to repeated episodes of chest tightness and fatigue experienced 
for five years, concurrent shortness of breath, and swelling of the whole body experienced for five months. The patient had undergone DDD pacing therapy five years prior to admission. The clinical diagnosis was dilated cardiomyopathy and thirddegree atrioventricular (AV) block. After pacemaker implantation, the patient's cardiac function was still class III. The electrocardiogram revealed sinus rhythm, and the QRS time was $200 \mathrm{~ms}$. Echocardiography revealed that the LVEDD was $58 \mathrm{~mm}$, and the LVEF was $39 \%$. The cardiothoracic ratio was 0.67 . The patient was dependent on ventricular pacing and presented with cardiac dysfunction, indicating that CRT was necessary. Subclavian vein angiography revealed complete occlusion from the left subclavian vein to the inferior vena cava. Therefore, we performed transthoracic epicardial lead implantation.

After the induction of general anesthesia, the patient was placed in the supine position, and the left chest was elevated. An incision of approximately $5 \mathrm{~cm}$ in length was created in the left anterolateral chest (Figure 1). The chest was accessed through the fourth intercostal space using a mini-thoracotomy retractor system. A longitudinal incision was created in the anterior pericardium, anterior to the left phrenic nerve to expose the heart. Unlike the first patient, two 6-0 Prolene sutures were placed in the triangular region located inferior to the left atrioventricular groove and superior to the first obtuse marginal branch of the left circumflex artery, and the epicardial lead was fixed in this avascular zone (Figure 3). The pacing parameters were measured. The left ventricular impedance was $640 \Omega$; threshold, $1.5 \mathrm{~V}$; and R-wave amplitude, $5.0 \mathrm{mV}$. A small incision was created in the corresponding part of the pericardium to remove the epicardial lead line, which was then pulled through the upper intercostal space into the subcutaneous tunnel and brought into the pocket of the pacemaker. The pacing parameters were measured again. The left ventricular impedance was $640 \Omega$; threshold, $1.5 \mathrm{~V}$; and R-wave amplitude, was $4.8 \mathrm{mV}$. A fistula was created in the pericardium posterior to the left phrenic nerve for drainage. Intermittent suturing was performed for the pericardial incision, and the chest was closed.

Case III: A 57-year-old female patient was admitted owing to repeated chest tightness and shortness of breath for experienced for more than six years. The patient had undergone three-chamber permanent pacemaker implantation three months prior to admission. The clinical diagnosis was dilated cardiomyopathy after CRT implantation with lead microdislocation. The cardiac function was class II-III. The electrocardiogram revealed sinus rhythm, and the QRS time was 198 ms. Echocardiography revealed that the LVEDD was $68 \mathrm{~mm}$, and the LVEF was $35 \%$. The cardiothoracic ratio was 0.66 . Given that micro-dislocation of the left ventricular lead was observed during pacing control, and the pacing threshold was high, we performed transthoracic epicardial lead implantation.

The surgical procedure was the same as that described for case II. The pacing parameters were measured. The left ventricular impedance was $560 \Omega$; threshold, $1.0 \mathrm{~V}$; and R-wave amplitude, $3.5 \mathrm{mV}$. After the epicardial lead line was removed from the pericardium and placed into the pocket of the pacemaker, the measured left ventricular impedance was 540 $\Omega$; threshold, $1.0 \mathrm{~V}$; and R-wave amplitude, $3.0 \mathrm{mV}$. A fistula was created in the pericardium posterior to the left phrenic nerve for drainage. Intermittent suturing was performed for the pericardial incision, and the chest was closed.

Case IV: A 57-year-old female patient was admitted owing to repeated episodes of chest tightness and shortness of breath experienced for more than two years, which had been aggravated for one week prior. The patient was diagnosed with dilated cardiomyopathy and complete left bundle branch block. The cardiac function was class III. An electrocardiogram revealed complete left bundle branch block, and the QRS time was 125 ms. Echocardiography revealed that the LVEDD was $75 \mathrm{~mm}$, and the LVEF was $28 \%$. The cardiothoracic ratio was 0.77 . The ejection fraction for non-ischemic dilated cardiomyopathy was $\leq 35 \%$, and the patient had New York Heart Association class III symptoms. Transvenous implantation of the left ventricular lead was very difficult; therefore, we performed epicardial lead implantation.

The surgical procedure was the same as described for case II. The pacing parameters were measured after fixing the lead. The left ventricular impedance was $520 \Omega$; threshold, $0.8 \mathrm{~V}$; and R-wave amplitude, $4.5 \mathrm{mV}$. Later, the pacing parameters were measured after the lead line was placed in the pacemaker pocket. The left ventricular impedance was $500 \Omega$; threshold, $0.9 \mathrm{~V}$; and R-wave amplitude, $3.5 \mathrm{mV}$.

\section{RESULTS}

Epicardial lead implantation successfully was performed in all four patients. The operative times were $135,60,70$, and $60 \mathrm{~min}$, respectively. There were no serious complications. Enalapril maleate, metoprolol or bisoprolol, and furosemide tablets were administered after the surgery, and antibiotics were administered to prevent infection.

Serious complications were not observed. The symptoms had improved significantly at two weeks after the surgery. Echocardiography revealed that the left ventricular enddiastolic diameter reduced from $83 \mathrm{~mm}$ to $67 \mathrm{~mm}$. After the programmable pacemaker was optimized, echocardiography revealed an improvement in synchronization between the interventricular and left ventricular contractions. The LVEF and cardiothoracic ratio, which were calculated using Simpson's rule, significantly improved. The Simpson's method is used to measure the left ventricular end-diastolic volume in the four- and two-chamber views of the apex of the heart using the color Doppler trackball; the end-systolic volume is measured, and the LVEF of patients with heart failure is calculated through the change in the area. The patient was discharged eight days after the surgery, and no obvious heart failure symptoms were observed.

In the first patient, the symptoms of phrenic nerve stimulation significantly were improved after adjusting the electrode position. In cases I, II, III, and IV, symptoms, such as chest tightness and shortness of breath, improved significantly after surgery. The range of the patients' blood pressure and heart rate was $90-98 / 50-64 \mathrm{mmHg}$ and $60-75$ beats per minute, 
respectively, and arrhythmias were not noted. Swelling in both limbs disappeared at 12,11, 4, and 7 days after the operation in cases I, II, III, and IV, respectively, and all patients were discharged. Echocardiography revealed a decrease in the left ventricular end-diastolic and end-systolic diameters. The LVEF for cases I, II, III, and IV was $38 \%, 48 \%, 43 \%$, and $49 \%$, respectively. Moderate-to-severe mitral regurgitation decreased to mild or moderate regurgitation, and severe tricuspid regurgitation decreased to moderate regurgitation. At 10 days after the surgery, the threshold of the epicardial lead was $<1.5 \mathrm{mV}$, which stabilized at $0.5-0.8 \mathrm{mV}$ during the follow-up visits.

\section{DISCUSSION}

CRT plays an important role in the treatment of chronic congestive heart failure [Separham 2019; Hoosain 2017]. It can change the sequence of myocardial activation via left ventricular pacing or biventricular pacing, thereby, improving the LVEF and hemodynamic parameters [Varma 2019; Vereckei 2018]. Many methods have been developed for placing the pacing leads. Among them, transvenous left ventricular lead implantation is the most common method. However, implantation failure may occur in as many as $10-30 \%$ of the patients because of malformation of the coronary sinus and target vein. Therefore, transthoracic epicardial pacing is an excellent therapeutic option in clinical practice.

As the coronary sinus and target vein were occluded, all our patients underwent epicardial lead implantation through a small transthoracic incision. Huntley et al. and Perrin et al reported that the outcomes of reasonable epicardial lead implantation are similar to those of endocardial lead implantation [Huntley 2018; Perrin 2018].

Based on our experience, there are two key aspects of this procedure. First, the location of the left ventricular epicardial lead is very important. In case I, tissue Doppler imaging was performed before the surgery and revealed the loss of synchronization between the base of the interventricular septum and the side wall of the left ventricle. The maximal difference in the time to peak was $251 \mathrm{~ms}$, which suggests that the side wall of the left ventricle was the area being excited last. After the lead was placed in the side wall of the left ventricle, Doppler imaging revealed that the difference in the time to peak was only $10 \mathrm{~ms}$, which implies improvement in left ventricular dyssynchrony. In case I, the lead was placed in the epicardium inferior to the first diagonal branch of the left anterior descending artery. The location was relatively lower, and the pacing threshold was relatively higher. Moreover, the lead was placed close to the phrenic nerve, thereby, causing phrenic nerve irritation. Diaphragm stimulation is a common complication after administration of CRT. The incidence of diaphragm stimulation during or after the administration of CRT reportedly is $1.6-3.0 \%$. Patients often experience seasonal discomfort, hiccups, decreased appetite, difficulty falling asleep, mood swings, and heart failure symptoms, which greatly affect their quality of life. Gurevitz reported that among 92 patients undergoing CRT, treatment was terminated for $4 \%$ of the patients because of phrenic nerve irritation [Gurevitz 2005]. Biffi et al. reported that the incidence of phrenic nerve irritation is $18-30 \%$ during CRT and that this irritation can be prevented by phrenic nerve isolation [Biffi 2011]. Champagne et al. reported that lead replacement can correct $77 \%$ of the side effects after phrenic nerve irritation [Champagne 2011]. In clinical practice, downregulation of the left ventricular output voltage or multi-polar lead placement may prevent phrenic nerve irritation [Bhaskaran 2019; Ohlow 2013]. In cases II, III, and IV, we adjusted the site of lead implantation, according to our experience with case I. The lead was placed in the triangular zone inferior to the left atrioventricular groove and superior to the first obtuse branch of the left circumflex artery. The pacing threshold in these three cases decreased compared with case I, and phrenic nerve irritation did not occur.

From our experience, we should perform an appropriate test during the operation and choose a suitable location. For example, the epicardial electrode lead can be implanted in the target site of the left or right lateral wall of the left ventricle or the target site of the left and right lateral walls. Timely program control and pacemaker voltage reduction effectively can reduce phrenic nerve stimulation. A review of the relevant literature revealed that resetting the electrode or performing phrenic nerve isolation can improve phrenic nerve stimulation in some patients with intractable phrenic nerve stimulation signs [Edgerton 2007].

The second key point is that it is critical to reduce surgical trauma and shorten the operative time. In the current study, we created a left-sided small incision in the fourth or fifth intercostal space and shortened the length of the incision to $5 \mathrm{~cm}$ using minimally invasive surgical devices; thus, excellent exposure was achieved for easy manipulation with less surgical trauma. However, this procedure may not be suitable for all patients. Nelson et al found that thoracoscopic left heart electrode implantation could not be performed in patients with severe chest adhesions. Open thoracotomy with epicardial electrode implantation was a feasible option for these patients [Nelson 2013].

In conclusion, during CRT, epicardial lead implantation via minimally invasive left-sided thoracotomy is safe, feasible, and effective. Our findings corroborate those of Marini et al, who reported a similar length of hospital stay, complication rate, and improvement in ventricular reverse remodeling and functional status between patients undergoing epicardial placement of the left ventricular lead using a video-assisted thoracoscopic technique and those undergoing the standard transvenous technique [Marini 2020].

Therefore, a hybrid surgery, combining interventional endocardial lead implantation with mini-thoracotomy, is an effective alternative method that can maximize the outcomes of CRT.

\section{ACKNOWLEDGEMENTS}

This research was funded by the Jiangxi Provincial Natural Science Foundation Project (20171BBG70067, 20181074, 
2016YNQN12036). This is work was supported by National Natural Science Foundation of China (\#81160019, \#81360031).

\section{REFERENCES}

Bhaskaran A, Tung R, Stevenson WG, et al. 2019. Catheter Ablation of VT in Non-Ischaemic Cardiomyopathies: Endocardial, Epicardial and Intramural Approaches [J]. Heart Lung Circ 28(1):84-101.

Biffi M, Boriani G. 2011. Phrenic stimulation management in CRT patients: are we there yet? [J]. Curr Opin Cardiol 26(1):12-16.

Brennan EJ. 2018. Chronic heart failure nursing: integrated multidisciplinary care [J]. Br J Nurs 27(12):681-688.

Champagne J, Healey JS, Krahn AD, et al. 2011. The effect of electronic repositioning on left ventricular pacing and phrenic nerve stimulation [J]. Europace 13(3):409-415.

Drugs for chronic heart failure [J]. 2019. Med Lett Drugs Ther 61(1569):49-54.

Edgerton JR, Edgerton ZJ, Mack MJ, et al. 2007. Ventricular epicardial lead placement for resynchronization by determination of paced depolarization intervals: technique and rationale [J]. Ann Thorac Surg 83(1):8992, 92.

Gurevitz O, Nof E, Carasso S, et al. 2005. Programmable multiple pacing configurations help to overcome high left ventricular pacing thresholds and avoid phrenic nerve stimulation [J]. Pacing Clin Electrophysiol 28(12):1255-1259.

Harjola VP, Parissis J, Brunner-La R H, et al. 2018. Comprehensive inhospital monitoring in acute heart failure: applications for clinical practice and future directions for research. A statement from the Acute Heart Failure Committee of the Heart Failure Association (HFA) of the European Society of Cardiology (ESC) [J]. Eur J Heart Fail 20(7):1081-1099.

Hoosain J, Whittier J, Hasni F, et al. 2017. The Initial Evaluation and
Management of a Patient with Heart Failure [J]. Curr Cardiol Rep 19(10):103.

Huntley GD, Deshmukh AJ, Warnes CA, et al. 2018. Longitudinal Outcomes of Epicardial and Endocardial Pacemaker Leads in the Adult Fontan Patient [J]. Pediatr Cardiol 39(7):1476-1483.

Konstantinides SV, Meyer G, Becattini C, et al. 2020. 2019 ESC Guidelines for the diagnosis and management of acute pulmonary embolism developed in collaboration with the European Respiratory Society (ERS) [J]. Eur Heart J 41(4):543-603.

Marini M, Branzoli S, Moggio P, et al. 2020. Epicardial left ventricular lead implantation in cardiac resynchronization therapy patients via a video-assisted thoracoscopic technique: Long-term outcome [J]. Clin Cardiol 43(3):284-290.

Nelson KE, Bates MG, Turley AJ, et al. 2013. Video-assisted thoracoscopic left ventricular pacing in patients with and without previous sternotomy [J]. Ann Thorac Surg 95(3):907-913.

Ohlow MA, Lauer B, Brunelli M, et al. 2013. The Use of a Quadripolar Left Ventricular Lead Increases Successful Implantation Rates in Patients with Phrenic Nerve Stimulation and/or High Pacing Thresholds Undergoing Cardiac Resynchronisation Therapy with Conventional Bipolar Leads [J]. Indian Pacing Electrophysiol J 13(2):58-65.

Perrin T, Maille B, Lemoine C, et al. 2018. Comparison of epicardial vs. endocardial reimplantation in pacemaker-dependent patients with device infection [J]. Europace 20(4):e42-e50

Separham A, Pourafkari L, Kazemi B, et al. 2019. Vitamin D deficiency and functional response to CRT in heart failure patients [J]. Herz 44(2):147-154.

Varma N, Boehmer J, Bhargava K, et al. 2019. Evaluation, Management, and Outcomes of Patients Poorly Responsive to Cardiac Resynchronization Device Therapy [J]. J Am Coll Cardiol 74(21):2588-2603.

Vereckei A, Szelenyi Z, Kutyifa V, et al. 2018. Novel electrocardiographic dyssynchrony criteria improve patient selection for cardiac resynchronization therapy [J]. Europace 20(1):97-103. 